See Article page 585.

\section{Commentary: Elusive right ventricle, do we know thee? Today a little more}

\author{
Danny Ramzy, MD, PhD
}

Right ventricle (RV) dysfunction presents unique challenges in patients - especially those immediately undergoing cardiac surgery. RV dysfunction and failure are formidable problems. Because of its distinct complexities, a failed RV is challenging to manage and well known to confer an increased risk of morbidity and mortality. ${ }^{1-6}$ Therefore, an effort to elucidate the details of patient characteristics and surgical variables resulting in $\mathrm{RV}$ dysfunction may lead to strategies reducing RV failure, possibly limiting morbidly and mortality following cardiac surgery.

Lang and colleagues ${ }^{7}$ broaden our knowledge of perioperative RV function. In a previous report, Singh and colleagues ${ }^{4}$ demonstrated that $\mathrm{RV}$ function was reduced following cardiac surgery, regardless of surgical procedure, approach, or reoperative status. The study by Singh and colleagues ${ }^{4}$ did not explore mechanisms beyond simple speculation, but it did likely serve as a catalyst to the work by Lang and colleagues. ${ }^{7}$ In the prospective observational study by Lang and colleagues, ${ }^{7} 109$ patients undergoing cardiac surgery are examined in an attempt to determine the intraoperative factors affecting RV function as assessed by transthoracic echocardiography. More specifically, they utilize machine learning to determine which factors have notable weight in their ability to predict RV dysfunction. This approach limits biased assessment and curtails erroneous conclusions. Lang and colleagues ${ }^{7}$ use tricuspid annular plane systolic excursion, RV longitudinal strain, and fractional area change to determine RV function. Echocardiographic RV functional assessment can be particularly

\footnotetext{
From the Department of Cardiac Surgery, Cedars-Sinai Medical Center, Los Angeles, Calif.

Disclosures: The author reported no conflicts of interest.

The Journal policy requires editors and reviewers to disclose conflicts of interest and to decline handling or reviewing manuscripts for which they may have a conflict of interest. The editors and reviewers of this article have no conflicts of interest.

Received for publication Nov 17, 2020; revisions received Nov 17, 2020; accepted for publication Nov 18, 2020; available ahead of print Nov 28, 2020.

Address for reprints: Danny Ramzy, MD, PhD, Department of Cardiac Surgery, Cedars-Sinai Medical Center, 127 S San Vicente Blvd, Suite A3105, Los Angeles, CA 90048 (E-mail: danny.ramzy@cshs.org)

J Thorac Cardiovasc Surg 2022;164:596-7

$0022-5223 / \$ 36.00$

Copyright $($ C 2020 by The American Association for Thoracic Surgery

https://doi.org/10.1016/j.jtcvs.2020.11.091
}
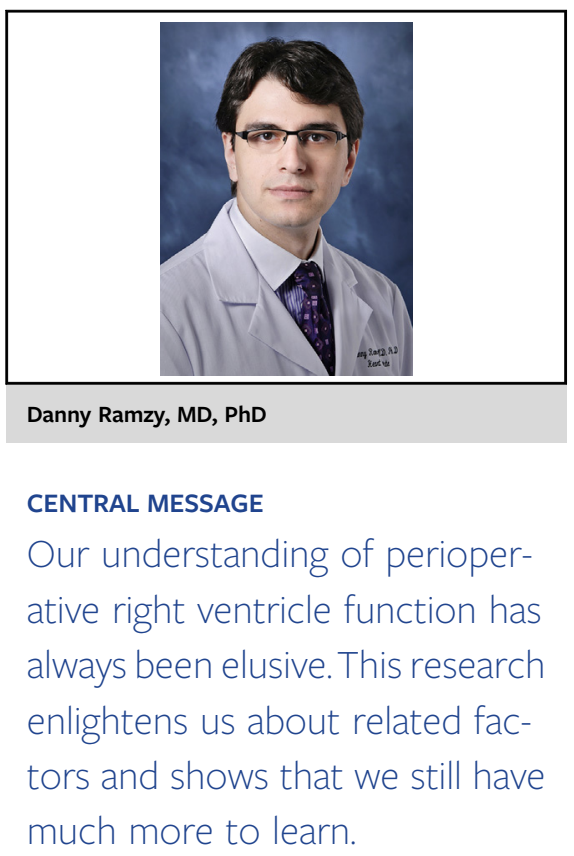

challenging, and no single specific modality is reliable. Therefore, a comparison of differing modalities may be more instructive. The American Society of Echocardiography strongly recommends routine use of at least 1 of these parameters to measure RV systolic function. ${ }^{8}$ To the authors' credit, they assess all 3 of these parameters, increasing the strength and validity of their study. The authors should be further commended because this study examines 33 variables and performs a remarkable statistical analysis. The resulting study has significant strengths related to scientific methodology in general. I recommend examining this work not only for its findings pertaining to RV function, but also for a broader view of how cardiac surgery-related statistical analysis should be performed and explained in an array of circumstances.

Notably, out of 33 variables, Lang and colleagues 7 sharply indicate that prolonged aortic crossclamp time, as well as reduced baseline RV function, are associated with inferior postoperative RV function. Coupling the aortic crossclamp time data with the significant importance of baseline RV function, we can conclude that the cardiac surgical community must be wary of operating on patients with reduced baseline RV function requiring an especially long procedural crossclamp time. Neither I nor the authors are suggesting that we should not operate on such patients. But the data strongly suggest that we consider optimizing these patients before surgery, and/or utilizing crossclamplimiting strategies. Possibilities include performing an on-pump beating surgery for coronary artery bypass 
grafting or performing tricuspid repair/replacement with the clamp off in patients with reduced RV function.

An important question most of the readership may share is whether the cardioplegia strategy will affect RV myocardial protection. Unfortunately, this study does not conclusively address whether different cardioplegia strategies have varying effects on RV function. Readers might object, of course, and claim that a slight improvement exists in 1 echocardiographic parameter (ie, tricuspid annular plane systolic excursion). However, in response, the study found that the choice of cardioplegia, as well as cardioplegia delivery strategies, was not associated with any clinically significant decrease in RV function as measured by all 3 parameters. In addition, the effect of topical cooling, with either ice or a cooling jacket, was not assessed because none of the surgeons in this study use this strategy. Therefore, this study provides no information as to whether a commonly utilized strategy has any effect on RV protection.

This study also highlights that the classic parameters that we as a surgical community monitor for RV function, such as central venous pressure (CVP), amount of tricuspid regurgitation (TR), or even reduced left ventricle function, do not correlate with worsening RV function. This may explain why, at times, it seems mysterious that our patients with normal CVP and TR have worsening RV function, whereas others with elevated CVP and worsening TR have more preserved RV function. It now seems that those classic parameters are not as reliable as we once believed.

Unfortunately, some significant parameters were not factored into their model. One unconsidered aspect is pulmonary artery (PA) pressure. PA pressure, more specifically PA hypertension, is a risk factor for RV failure and can potentially be an independent predictor. Despite this limitation, Lang and colleagues ${ }^{7}$ provide a significant breadth of information regarding most known operative predictors to help readers avoid, limit, or prevent RV dysfunction. Although RV failure is an important source of morbidity and mortality after certain types of cardiac surgery, the elucidation of the predictors of RV failure alone is not sufficient. This information must lead to the development of strategies to manage this problem. Lang and colleagues ${ }^{7}$ suggest that efforts be made to minimize aortic crossclamp time, to optimize preoperative RV function, and that improved myocardial protection strategies may improve RV function following cardiac surgery. However, the concern that I raise is that the relationship between predictor variables of RV function and postoperative outcomes were not investigated, which leaves readers with the speculation that predictor modification would result in improved RV dysfunction and outcomes. Despite this reservation, I hope that predictor modification will translate into improved outcomes resulting in lower morbidity and mortality.

RV failure after cardiac surgery is an uncommon but potentially catastrophic complication. The key to any successful treatment strategy is the identification of patient or surgical characteristics that portend the greatest risk of RV dysfunction. Such knowledge will heighten surgeons' suspicion for dysfunction perioperatively and aid in making vital perioperative decisions or strategy changes to limit the development of RV failure. Lang and colleagues ${ }^{7}$ observations and conclusions bring us 1 step closer to developing algorithms and treatment protocols to limit the potentially deleterious outcome of RV failure. The most vital conclusion is that we must start utilizing data-driven strategies to improve our outcomes. If we lose sight of this valuable lesson, we will keep repeating the same mistakes and our patients may ultimately pay the price in morbidity and mortality.

\section{References}

1. Haddad F, Couture P, Tousignant C, Denault AY. The right ventricle in cardiac surgery, a perioperative perspective: II. Pathophysiology, clinical importance, and management. Anesth Analg. 2009;108:422-33.

2. Algarni KD, Arafat A, Algarni AD, Alfonso JJ, Alhossan A, Elsayed A, et al. Degree of right ventricular dysfunction dictates outcomes after tricuspid valve repair concomitant with left-side valve surgery. Gen Thorac Cardiovasc Surg. November 6, 2020 [Epub ahead of print].

3. Bootsma IT, de Lange F, Koopmans M, Haenen J, Boonstra PW, Symersky T, et al Right ventricular function after cardiac surgery is a strong independent predictor for long-term mortality. J Cardiothorac Vasc Anesth. 2017;31:1656-62.

4. Singh A, Huang X, Dai L, Wyler D, Alfirevic A, Blackstone EH, et al. Right ventricular function is reduced during cardiac surgery independent of procedural characteristics, preoperative status, or pericardiotomy. J Thorac Cardiovasc Surg. 2020;159:1430-8.

5. Vlahakes GJ. Right ventricular failure after cardiac surgery. Cardiol Clin. 2012 30:283-9.

6. Itagaki S, Hosseinian L, Varghese R. Right ventricular failure after cardiac surgery: management strategies. Semin Thorac Cardiovasc Surg. 2012;24:188-94.

7. Lang AL, Huang X, Alfirevic A, Blackstone E, Pettersson G, Singh A, et al. Patient characteristics and surgical variables associated with intraoperative reduced right ventricular function. J Thorac Cardiovasc Surg. 2022;164:585-95.e5.

8. Rudski LG, Lai WW, Afilalo J, Hua L, Handschumacher MD, Chandrasekaran K, et al. Guidelines for the echocardiographic assessment of the right heart in adults: a report from the American Society of Echocardiography endorsed by the European Association of Echocardiography, a registered branch of the European Society of Cardiology, and the Canadian Society of Echocardiography. J Am Soc Echocardiogr. 2010;23:685-713. 